\title{
Overview of Scene Change Detection - Application to Watermarking
}

\author{
Dolley Shukla \\ Associate Professor, Dept. of IT \\ Shri Shankaracharya College of Engg .\& Tech.
}

\author{
Manisha Sharma \\ Professor \& HoD. E \& Tc \\ Bhilai Institute of Technology, Durg
}

\begin{abstract}
With the advent of digital video and digital broadcasting, watermarking of video data has been one of the important issues. Scene change detection (SCD) is one of several fundamental problems in the design of video watermarking. It is the first step towards automatic segmentation, annotation, and indexing of video data. SCD is also used in other aspects of watermarking i.e. copy-protection, copyright-protection of videos. Therefore, for the copy-protection of video using watermarking, scene change detection is an important step. In this paper we provide classification and comparison of different scene change detection techniques \& algorithms.
\end{abstract}

\section{Keywords}

Video watermarking, abrupt change, scene change detection, compressed \& uncompressed video

\section{INTRODUCTION}

Detection of scene changes play important roles in video processing with many applications ranging from watermarking, video indexing, video summarization to object tracking and video content management. Scene change detection is an operation that divides video data into physical shots[1]. Over the last three decades, scene change detection has been widely studied and researched. As a result, many scene change detection techniques have been proposed and published in the literature. For our convenience of surveying existing research in this subject area, all these algorithms and techniques can be broadly classified as operating on decompressed data (pixel domain), or working directly on the compressed data (compressed domain)[2]. Scene changes are divided into two types: Abrupt scene change and Gradual scene change. Abrupt scene changes result from editing "cuts" and detecting them is called cut detection either by colour histogram comparison on the uncompressed video or by DCT coefficient comparison. Gradual scene changes result from chromatic edits, spatial edits and combined edits. Gradual scene changes include special effects like zoom, camera pan, dissolve and fade in/out, etc [3].

\section{FEATURES OF SCENE BOUNDARY DETECTION}

Almost all scene change detection algorithms reduce the large dimensionality of the video domain by extracting a small number of features from each video frame. These are extracted either from the whole frame or from a subset of it, which is called a region of interest (ROI).

Such features include[4]:

\subsection{Luminance/colour}

The simplest feature that can be used to characterize a ROI is its average gray scale luminance. This, however, is susceptible to illumination changes. A better choice is to use some statistics of the values in a colour space.

\subsection{Luminance/colour histogram}

A richer feature for a ROI is the gray scale or colours histogram. It is quite discriminates, easy to compute and mostly insensitive to translational, rotational and zooming camera motion, for the above reasons it is widely used.

\subsection{Image edges}

An obvious choice of feature is edge information in a ROI. Edges can be used as is, be combined into objects or used to extract ROI statistics. They are invariant to illumination changes and most motion, and they correspond somewhat to the human visual perception. Their main disadvantage is computational cost, noise sensitivity and high dimensionality.

\subsection{Transform coefficients (DFT, DCT, Wavelet):}

These are a classic way to describe the texture of a ROI. The DCT coefficients are also present in MPEG encoded video streams or files. Their greatest problem is that they are generally not invariant to camera zoom.

\subsection{Other features}

A number of other features are used in the literature, such as the colour angiogram.

\subsection{Multiple features}

Many algorithms extract several types of features either to use them in combination or for subsequent processing and analysis.

\section{REVIEW ON SCENE CHANGE DETECTION}

With the rapid growth of multimedia production and distribution, more and more people, concern the problem on copy protection of multimedia data. This problem become obvious as the explosive growth of the internet in recent few years. Video watermarking requires the scene change detection in the first step. Hence, this section presents a review of different scene change detection techniques of videos. Scene change detection algorithms are based on the pixel differences, compressed(MPEG2)domains, temporal segmentation luminance histograms based framework for temporal segmentation, sudden scene change detection for MPEG-2 compressed video, algorithm using direct edge information extraction from MPEG video data is used. 
The comparison and analysis of scene change detection algorithms is described in the following subsections.

Lock Yeo and Bede Liu et al proposed scene analysis which is in the form of temporal segmentation of videos and flashlight detection can be performed on compressed videos using only the dc coefficients of compressed domain [5].The core information of videos is captured in the greatly reduced $\mathrm{dc}$ sequences, thus permitting these operations to be performed much more efficiently. But these temporal segmentation is done only on compressed video, not for uncompressed video.

$\mathrm{K}$. Tse et al presents scene change detection algorithms which is based on the pixel differences and compressed(MPEG-2) domains[6].The main problem of this method is that it suffers from the variations incurred by camera motion. This algorithm has the potential to detect gradual scene changes.

Haitao Jiang et al presents a Scene change detection Techniques for video database system which provide automatic segmentation, annotation, and indexing of video data[7].It provide a taxonomy that classifies the existing algorithms into three categories: full video image based, compressed-video-based and model-based algorithms.

P.Bouthemy et al gives Approach to scene change detection \& characterization [8].The Method relay on statistical technique robustness and efficiency for compressed video. The Image is represented in 2D affine model.

Xinying Wang et al suggested a twice difference of luminance histograms based framework for temporal segmentation[9].The method has been introduced to perform unsupervised.However, this method need to be further expended to detect complex transitions between scene change as fades and dissolve.

Seong-Whan Lee has presented a method for scene change detection algorithm using direct edge information extraction from MPEG video data[10]. The paper proposed a fast scene change detection algorithm using direct feature extraction from MPEG compressed videos, and evaluate this technique using sample video data.This process was made possible by a new mathematical formulation for deriving the edge information directly from the discrete cosine transform coefficients.

W. A. C. Fernando proposed an algorithm for sudden scene change detection for MPEG-2 compressed video[11].This uses the number of interpolated macroblocks in B-frames to identify the sudden changes. This algorithm can detect abrupt scene changes irrespective of the nature of the sequences.

Shu-Ching Chen1 et al[1]proposed a technique for uncompressed video data.The paper compare the segmentation mask maps between two successive video frames \& Cannot be used for compressed video.

Anastasios Dimou et alproposed a Scene change detection for H.264[12].It scribes the correlation between local statistical characteristics,scene duration and scene change and It uses only previous frames for the detection.

Priyadarshinee Adhikari et al proposed a segmentation method based on colour difference histogram[13].The paper describes a scene change detection method by scaling the histogram difference between the two frames. It provide the scaled frame difference that is dynamically compressed by log formula and it is more convenient to decide the threshold. It is based on detecting edges in two neighbouring images and comparing these images.

Purnima.S.Mittalkod et al present a paper in which it gives the classification of shot boundary detection algorithms, including those that deal with gradual shot transitions[4].This paper compares different Shot Boundary Detection Algorithms and Techniques \& concluded that these techniques can be further refined and implemented for automatic shot detection. However, video analysis remains a challenging task with respect to unstructured home videos.

\section{COMPARATIVE ANALYSIS}

This section gives the comparative analysis of different researchers on scene change detection algorithms on the basis of the area, limitations and their performance. The performance is given in terms of parameters precision \& recall .

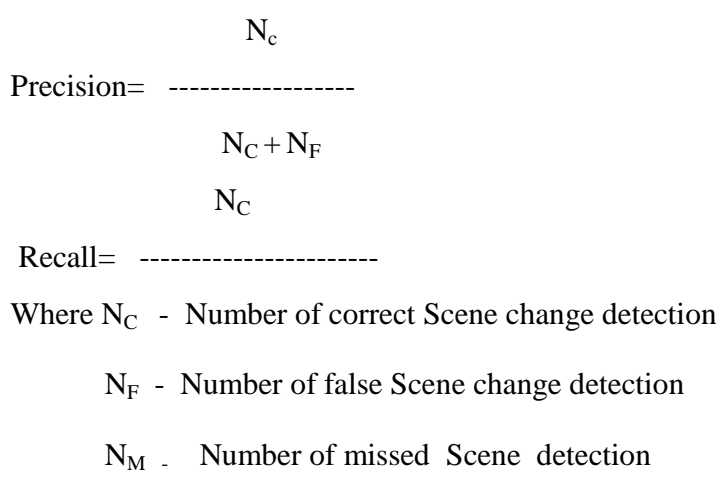

$\mathrm{N}_{\mathrm{C}}+\mathrm{N}_{\mathrm{M}}$ 
Table 1 . Comparative study of different researchers on scene change detection algorithms

\begin{tabular}{|c|c|c|c|c|c|c|c|}
\hline $\begin{array}{l}\text { S. } \\
\mathbf{N} \\
\text { o. }\end{array}$ & Year & Author & Area & Detail & Limitation & $\begin{array}{l}\text { Precis } \\
\text { ion }\end{array}$ & Recall \\
\hline 1. & 1995 & $\begin{array}{l}\text { Lock } \\
\text { Yeo et } \\
\text { al[5] }\end{array}$ & $\begin{array}{l}\text { compressed } \\
\text { videos }\end{array}$ & $\begin{array}{l}\text { - Temporal segmentation of } \\
\text { videos and flashlight } \\
\text { detection }\end{array}$ & $\begin{array}{l}\text { Done only on compressed } \\
\text { video, not for uncompressed } \\
\text { video. }\end{array}$ & $99 \%$ & $92.6 \%$ \\
\hline 2. & 1995 & $\begin{array}{l}\text { K. Tse } \\
\text { et al [6] }\end{array}$ & $\begin{array}{l}\text { compressed( } \\
\text { MPEG-2) data }\end{array}$ & $\begin{array}{l}\text { - } \begin{array}{l}\text { Based on the pixel } \\
\text { differences }\end{array} \\
\text { - Potential to detect gradual } \\
\text { scene changes }\end{array}$ & $\begin{array}{l}\text { Suffers from the variations } \\
\text { incurred by camera motion }\end{array}$ & - & - \\
\hline 3. & 1998 & $\begin{array}{l}\text { Haitao } \\
\text { Jiang et } \\
\text { al [7] }\end{array}$ & $\begin{array}{l}\text { Compressed } \\
\text { video for } \\
\text { video } \\
\text { database } \\
\text { systems. }\end{array}$ & $\begin{array}{l}\text { Provide automatic } \\
\text { segmentation, annotation, } \\
\text { and indexing of video data. }\end{array}$ & $\begin{array}{l}\text { Video-coding and decoding } \\
\text { schemes are difficult. }\end{array}$ & $90 \%$ & $90 \%$ \\
\hline 4. & 1999 & $\begin{array}{l}\text { P.Bouth } \\
\text { emy et } \\
\text { al [8] }\end{array}$ & $\begin{array}{l}\text { Compressed } \\
\text { video }\end{array}$ & $\begin{array}{l}\text { - Method relay on statistical } \\
\text { technique robustness and } \\
\text { efficiency }\end{array}$ & $\begin{array}{l}\text { Image represented in } 2 \mathrm{D} \text { affine } \\
\text { model }\end{array}$ & - & - \\
\hline 5. & 2000 & $\begin{array}{l}\text { Xinying } \\
\text { Wang' } \\
\text { et al[9] }\end{array}$ & $\begin{array}{l}\text { Unsupervised[ } \\
\text { 3]. }\end{array}$ & $\begin{array}{l}\text { - Twice difference of } \\
\text { luminance histograms } \\
\text { based framework for } \\
\text { temporal segmentation. }\end{array}$ & $\begin{array}{l}\text { Method need to be further } \\
\text { expended to detect complex } \\
\text { transitions between scene } \\
\text { change as fades and dissolve. }\end{array}$ & - & - \\
\hline 6. & 2000 & $\begin{array}{l}\text { Seong- } \\
\text { Whan } \\
\text { Lee[10] }\end{array}$ & $\begin{array}{l}\text { MPEG video } \\
\text { data }\end{array}$ & $\begin{array}{l}\text { Edge information } \\
\text { extraction from MPEG } \\
\text { video data is used[8]. }\end{array}$ & $\begin{array}{l}\text { Still developing for gradual } \\
\text { scene detection methods. }\end{array}$ & - & - \\
\hline 7. & 2001 & $\begin{array}{l}\text { W. A. } \\
\text { C. } \\
\text { Fernand } \\
\text { o[11] }\end{array}$ & $\begin{array}{l}\text { MPEG-2 } \\
\text { compressed } \\
\text { video }\end{array}$ & $\begin{array}{l}\text { - Uses the number of } \\
\text { interpolated macroblocks in } \\
\text { B-frames to identify the } \\
\text { sudden changes } \\
\text { - Detect abrupt scene } \\
\text { changes irrespective of the } \\
\text { nature of the sequences. }\end{array}$ & $\begin{array}{l}\text { Algorithm can detect abrupt } \\
\text { scene changes irrespective of } \\
\text { the nature of the sequences. }\end{array}$ & - & - \\
\hline 8. & 2001 & $\begin{array}{l}\text { Shu- } \\
\text { Ching } \\
\text { Chen1 } \\
\text { et al[1] }\end{array}$ & $\begin{array}{l}\text { Uncompresse } \\
\text { d video data }\end{array}$ & $\begin{array}{l}\text { - Compare the segmentation } \\
\text { mask maps between two } \\
\text { successive video frames. }\end{array}$ & $\begin{array}{l}\text { Cannot be used for compressed } \\
\text { video. }\end{array}$ & $\begin{array}{l}\text { Above } \\
90 \%\end{array}$ & $\begin{array}{l}\text { Above } \\
90 \%\end{array}$ \\
\hline 9. & 2005 & $\begin{array}{l}\text { Anastasi } \\
\text { os } \\
\text { Dimou } \\
\text { et al[12] }\end{array}$ & H.264 video & $\begin{array}{l}\text { Provide the correlation } \\
\text { between local statistical } \\
\text { characteristics, rene } \\
\text { duration and scene change. }\end{array}$ & $\begin{array}{l}\text { It uses only previous frames } \\
\text { for the detection. }\end{array}$ & $97 \%$ & $97 \%$ \\
\hline $\begin{array}{l}\text { S. } \\
\mathbf{N} \\
\mathbf{o}\end{array}$ & Year & Author & Area & Detail & Limitation & $\begin{array}{l}\text { Precis } \\
\text { ion }\end{array}$ & Recall \\
\hline
\end{tabular}




\begin{tabular}{|c|c|c|c|c|c|c|c|}
\hline 10 & 2006 & $\begin{array}{l}\text { Z.Li. J. } \\
\text { Jiang }\end{array}$ & $\begin{array}{l}\text { Fast and real } \\
\text { time } \\
\text { Implementatio } \\
\mathrm{n}\end{array}$ & $\begin{array}{l}\text { - Uses Infra coded } \\
\text { macroblocks. } \\
\text { - } \begin{array}{l}\text { Improve precision \& recall } \\
\text { rate }\end{array} \\
\text { - Fast processing speed }\end{array}$ & Algorithmic complexity & $\begin{array}{l}75.49 \\
\%\end{array}$ & $\begin{array}{l}90.38 \\
\%\end{array}$ \\
\hline 11 & 2008 & $\begin{array}{l}\text { Priyadar } \\
\text { shinee } \\
\text { Adhikar } \\
\text { i et al } \\
\text { [13] }\end{array}$ & $\begin{array}{l}\text { Uncompresse } \\
\text { d video }\end{array}$ & $\begin{array}{l}\text { - Using one of the methods } \\
\text { of the colour histogram } \\
\text { wherein scaling of the } \\
\text { histogram metrics is an } \\
\text { added feature. }\end{array}$ & $\begin{array}{l}\text { Cannot be used for compressed } \\
\text { video. }\end{array}$ & $\begin{array}{l}90.47 \\
\%\end{array}$ & $\begin{array}{l}88.37 \\
\%\end{array}$ \\
\hline 12 & 2011 & $\begin{array}{l}\text { Purnima } \\
\text { S.Mittal } \\
\text { kod et } \\
\text { al [4] }\end{array}$ & Video Shot & $\begin{array}{l}\text { - Shot boundary detection } \\
\text { algorithms, including those } \\
\text { that deal with gradual shot } \\
\text { transitions }\end{array}$ & $\begin{array}{l}\text { Further refined and } \\
\text { implemented for automatic shot } \\
\text { detection. }\end{array}$ & - & - \\
\hline
\end{tabular}

\section{APPLICATIONS}

Important applications of change detection include video surveillance, remote sensing, Video Indexing, civil infrastructure, underwater sensing

5.1 Video Surveillance - A change detection module is the fundamental part of a common video-surveillance system. change detection step to find the objects present in the scene. Video surveillance systems are based on a given static configuration of one or more fixed cameras. Video Surveillance System is a motion or scene detection software that monitors your home, office, or any premise.

5.2 Remote Sensing - Remote Sensing along with digital image processing techniques helps in identifying change between two (or more) dates that is uncharacterised of normal variation. Remote Sensing helps in acquiring multi spectral spatial and temporal data through space borne remote sensors. Image processing technique helps in analyzing the dynamic changes associated with the earth resources such as land and water using remote sensing data.

5.3 Video Watermarking - Scene change detection in videos is a primary requirement of video processing applications used for the purpose of generating data needed by video data management systems Digital watermarking continues to remain the main technique used for this purpose.

5.4 Video Indexing - Video indexing involves "segmentation, analysis and abstraction" of video content. Content-based indexing and retrieval of video are important in many applications, and scene-change detection plays an essential role in these tasks.

5.5 Underwater Sensing - Underwater sensing which detect differences between linear combinations of the spectral bands from the two acquisitions.

\section{SCENE CHANGE DETECTION FOR WATERMARKING}

The success of the segmentation approach depends largely on how well the video materials are divided into segments or shots. A shot is defined as a part of the video that results from one continuous recording by a single camera. A scene is composed of a number of shots, while a television broadcast consists of a collection of scenes. The gap between two shots is called a shot boundary.

There are mainly four different types of common shot boundaries within shots. These are A cut , A fade, A dissolve $\&$ A wipe. There have been a number of various approaches to handle different shot boundaries[14].

- Shot Boundary Detection based on Color Diagrams

- $\quad$ Edge Detection

- Shot Boundary Detection Using Macroblocks

Therefore for the watermarking scheme, some scene change analysis were proposed,which were based on Abrupt scene change detection and were robust against the attacks of frame dropping, averaging and statistical analysis, but were not solved effectively in the past $[15,16,17]$.Moreover, a second approach is proposed by Kintu patel et al, which can improve the robustness of the watermarking scheme. In this sceme, A video and a watermark are taken as the input, and the color binary watermark is then decomposed into different components $(\mathrm{R}, \mathrm{G}, \mathrm{B})$ which are embedded in corresponding components of frames of the original color video. As applying a fixed image watermark to each frame in the video leads to the problem of maintaining statistical and perceptual invisibility, this scheme employs independent watermarks for successive but different scenes. However, applying independent watermarks to each frame also presents a problem if regions in each video frame remain little or no motion frame after frame. These motionless regions may be statistically compared or averaged to remove the independent watermarks [18]. Consequencely, it uses an identical watermark within each motionless scene. With these mechanisms, this method is robust against the attacks of frame dropping, averaging, swapping, and statistical analysis. The first approach tested at Dublin was a shot detection based on color histograms. They computed frame-to-frame similarities based on colors which appeared within them, albeit of the relative positions of those colors in the frame. After computing the inter-frame similarities, a threshold can be used to indicate shot boundaries. It needs dynamic threshold to work on other effects than simple shot boundaries[19].

To extract robust frame difference from consecutive frames, it uses verified $\mathrm{x} 2$ test which shows good performance 
comparing existing histogram based algorithm and to increase detection effect of color value subdivision work, color histogram comparison using the weight of brightness grade. Also to reduce the loss of spatial information and to solve the problem for two different frames to have similar histogram, we used local histogram comparison.

The scene-based watermarking scheme is robust against various attacks because it does not require original video as well as watermarked video for original video and watermark video recovery as it has used blind technique for watermarking according to scene change algorithm. This scheme gives the perfect solution for where to do watermarking in video thus it will become robust against every attack.

\section{CONCLUSION}

This paper reviews some basic techniques of scene change detection \& their comparative analysis. Scene change detection is one of the important initial step towards video watermarking. The performance of Scene Change Detection can be compared using Precision and Recall Parameters. The Comparative Analysis shows that the algorithm based on Abrupt scene change detection provides significant precision and Recall which is an advantage when will use in watermarking application It is concluded that video watermarking can be done using abrupt scene change detection which further improve the robustness of the watermarking scheme against various attack.

\section{REFERENCES}

[1] Shu-Ching Chen, Mei-Ling Shyu, Cheng-Cui Zhang, Kashyap, R. L.: 2001 Video Scene change Detection method using Unsupervised Segmentation and object Tracking, In: Multimedia and Expo,2001,ICME 2001,IEEE International Conference on, 22-25 AUG. 2001, pp. 56-59

[2] Li1,Z.,Jiang, j., Xiao, G., Fang,H. 2006 An Effective and Fast Scene Change Detection Algorithm for MPEG Compressed Videos, In: ICIAR 2006, LNCS 4141, pp. 206-214.

[3] Chong-Wah Ngo, Pong, Ting-Chuen., Zhang, HongJiang., Chin, R.T 2000 Motion-Based Video Representation for Scene Change Detection, In: Patttern Recognition,2000 proceedings $15^{\text {th }}$ International Conference on, 2000, , vol. 1, 2000, pp-827-830, )

[4] S.Mittalkod, Purnima., Srinivasan, G.N 2011 Shot Boundary Detection Algorithms and Techniques: A Review, In: Research Journal of Computer Systems Engineering- An International Journal, Vol. 2, Issue 2.

[5] Yeo, Boon-Lock., Liu, Bede 1995 Rapid Scene Analysis on Compressed Video, IEEE Transactions on Circuits \& Systems for VideoTechnology, vol. 5, No. 6.

[6] Tse, K., J. Wei, J., S. Panchanathan,S 1995 A Scene Change Detection Algorithm for MPEG Compressed Video Sequences, In: Electrical \& computer engineering,Canadian conference on, vol.02, pp.827-830
[7] Haitao Jiang,Abdelsalam Helai, Ahmed K. Almagarmid , Anupam Joshi, 1998 Scene change detection Techniques for video database systems, In: Multimedia Systems @ Springer Verilog, No.06, PP.186-195.

[8] Bouthemy,P., M.Gelgon, M., Ganansia,F. 1999 EIRISA,Rennes A unified approach to shot change detection and camera motion characterization, In: Circuits \& systems for Video Technology, IEEE Transactions on, vol.9, Issue.7, pp.1051-8251.

[9] Wang, Xinying., Zhengke VCTeng 2000 Scene Abrupt Change Detection , In: Electrical \& computer Engineering 2000,Canadian conference on,vol.2 , 2000,pp.880-883 .

[10] Lee , Seong -Whan , Kim, Young-Min , Choi, Sung Woo 2000 Fast Scene Change Detection using Direct Feature, Extraction from MPEG Compressed Videos, In: IEEE Transactions on multimedia,Dec. 2000, No. 2, issue.4, pp: $240-254$.

[11] W.A.C.Fernando, C.N.Canagarajah, Bull, D.R. 2001 Scene change detection algorithms for content based video indexing and retrieval , Electronics \& Communication Engineering Journal ,vol. 13, Issue: 3, pp.117-126.

[12] Anastasios Dimou, 2005 Scene Change Detection for H.264 Using Dynamic Threshold Techniques , In: Proceedings of 5th EURASIP Conference on Speech and Image Processing, Multimedia Communications and Service.

[13] Adhikari Priyadarshinee., Gargote, Neeta., Digge, Jyothi., B.G.Hogade, B.G. 2008 Abrupt Scene Change Detection, In: World Acadamy of Science, Engineering and Technology 42 2008, pp: 711-716.

[14] Gonzalez; Digital Image Processing 2/E. Prentice-Hall, Englewood Cliffs (2002).

[15] Lee, J., Jung,S. 2001 : A Survey Of Watermarking Techniques Applied To Multimedia, In: Proceedings 2001 IEEE International Symposium On Industrial Electronics (Isie2001), Vol. 1, pp. 272-277.

[16] Hwang, Ren-Junn. 2000 : A digital image copyright protection scheme based on visual cryptography, In: Tamkang Journal of Science and Engineering, vol. 3, no. 2, pp. 97-106.

[17] Houmansadr, Amir., Ghaemmaghami,Shahrokh.: A novel video watermarking method using visual cryptography, IEEE International Conference on Engineering of Intelligent Systems, Islamabad, Pakistan, (2006)

[18] Duan, F., King,I., Xu, L., Chan,L 1998 .: Intra-block max min algorithm for embedding robust digital watermark into BIBLIOGRAPHY 128 image, In: Horace H.S. Ip and Arnold W.M. Smeulders, editors, Proceedings of the IAPR International Workshop on Multimedia Information Analysis and Retrieval, MINAR' 98, Lecture Notes in Computer Science, Vol. 1464, pp. 255-264, Berlin Heidelberg, Germany, Springer- Verlag..

[19] N. Checcacci, N., Barni, M., Bartolini,F.., Basagni,S.: Robust video watermarking for wireless multimedia communications, In: Proceedings IEEEE Wireless Communications and Networking Confernce 2000, WCNC. 2000, Vol. 3, pp. 1530-1535. 